\title{
ON STRICTLY PSEUDOCONVEX DOMAINS WITH KÄHLER-EINSTEIN BERGMAN METRICS
}

\author{
Siqi Fu AND Bun Wong
}

\section{$\S 0$. Introduction}

For any bounded domain in $\mathbb{C}^{n}$, there exists a canonical Kähler metric-the Bergman metric. It follows from the work of Pflug $[\mathrm{P}]$ and Ohsawa [O] (see also the recent work of Diederich and Ohsawa $[\mathrm{D}-\mathrm{O}]$ ) that the Bergman metric is complete for any pseudoconvex domain with $C^{1}$-smooth boundary. On the other hand, it follows from the deep work of Cheng and Yau [C-Y] that for any $C^{2}$-smoothly bounded pseudoconvex domain, there exists a unique complete Kähler-Einstein metric with Ricci curvature -1. This result was later extended by Mok and Yau [M-Y] to an arbitrary bounded domain of holomorphy. So far, the metric constructed by Cheng and Yau has been the only known canonical metric that is complete for any bounded domain of holomorphy.

It is well-known that for any bounded homogeneous domain in $\mathbb{C}^{n}$, the Bergman metric has constant Ricci curvature -1 [B]. Thus the Bergman metric is identical to the Kähler-Einstein metric constructed by Cheng-Yau. In his famous problem list in differential geometry [Y, pp. 679], S.-T. Yau raised the following question (in a slightly different form): classify pseudoconvex domains whose Bergman metrics are Kähler-Einstein. It was conjectured by S.-Y. Cheng in his Taniguchi lecture in 1979 [C] that if the Bergman metric of a strictly pseudoconvex domain is Kähler-Einstein, then the domain is biholomorphic to the ball.

In this paper, we shall prove the following:

Main Theorem. Let $\Omega \subset \subset \mathbb{C}^{2}$ be a simply-connected strictly pseudoconvex domain with smooth boundary. If the Bergman metric of $\Omega$ is Kähler-Einstein, then $\Omega$ is biholomorphic to the ball.

Apparently, the above theorem is only the first step in understanding Yau's problem because the most difficult part of the problem is in the case of pseudoconvex domains with non-smooth boundaries. The proof of the main theorem is based on the fundamental work of Fefferman [Fe1] and intriguing results of Burns-Graham (see [G1]) and Chern-Ji [C-J]. This paper is organized as follows. In Sect.1, we recall some necessary definitions and give equivalent conditions to

Received May 9, 1997.

1991 Mathematics Subject Classification. Primary: 32H15; secondary 32F07, 32C10. 
the Kähler-Einstein condition on the Bergman metric. In Sect.2, we study Fefferman's asymptotic expansion of the Bergman kernel for a smoothly bounded strictly pseudoconvex domain in $\mathbb{C}^{n}$. We prove that if the Bergman metric is Kähler-Einstein, then the coefficient of the logarithmic term vanishes to infinite order at the boundary. The main theorem is then followed from above-mentioned results of Burns-Graham and Chern-Ji. Sect.3 contains further remarks and questions.

For information on the Bergman and Kähler-Einstein metrics on strictly pseudoconvex domains, we refer readers to the excellent exposition by C. Fefferman [BFG].

\section{$\S 1$. Preliminaries}

Let $\Omega$ be a bounded domain in $\mathbb{C}^{n}$ and let $K=K(z, z)$ be its Bergman kernel. The Bergman metric is a Kähler metric given by

$$
d s^{2}=\sum_{\alpha, \beta=1}^{n} g_{\alpha \bar{\beta}} d z^{\alpha} d \bar{z}^{\beta}
$$

where $g_{\alpha \bar{\beta}}=\partial^{2} \log K / \partial z_{\alpha} \partial \bar{z}_{\beta}$. Let $G(z)=\operatorname{det}\left(g_{\alpha \bar{\beta}}\right)$. Then the Ricci tensor of the Bergman metric is given by

$$
R_{\alpha \bar{\beta}}=-\frac{\partial^{2} \log G(z)}{\partial z_{\alpha} \partial \bar{z}_{\beta}} .
$$

The Bergman metric is Kähler-Einstein if $R_{\alpha \bar{\beta}}=c g_{\alpha \bar{\beta}}$ for some constant $c$. Since here we only concern about bounded domains in $\mathbb{C}^{n}$, it is well-known that the constant $c$ is necessary negative (see [C-Y, pp. 518 ]).

Let $B(z)=G(z) / K(z, z)$. It follows easily from the transformation formula for the Bergman kernel that the function $B(z)$ is invariant under biholomorphic mappings in the sense that if $\phi$ is a biholomorphic map from $\Omega_{1}$ to $\Omega_{2}$, then $B_{\Omega_{1}}(z)=B_{\Omega_{2}}(\phi(z))$. This function was introduced by Bergman and is called the Bergman invariant function [B]. Interesting application of the Bergman invariant function was given by Greene and Krantz [G-K]. Boundary behavior of invariants (including $B(z)$ ) related to the Bergman kernel was studied in [BSY] and [K-Y].

The following Monge-Ampére type equation was introduced by Fefferman [Fe2]:

$$
\begin{aligned}
J(u) \equiv(-1)^{n} \operatorname{det}\left(\begin{array}{cc}
u & u_{\bar{\beta}} \\
u_{\alpha} & u_{\alpha \bar{\beta}}
\end{array}\right)=1 & \text { in } \Omega, \\
u=0 & \text { at } b \Omega .
\end{aligned}
$$

Fefferman proved that a strictly pseudoconvex domain with smoothly boundary has a smooth defining function $\rho$ such that $J(\rho)=1+O\left(\rho^{n+1}\right)$. This approximate solution turned out to be a powerful tool in constructing invariants for 
strictly pseudoconvex domains $[\mathrm{G} 1,2]$. It was proved by Cheng-Yau $[\mathrm{C}-\mathrm{Y}]$ that the equation (MA) has a unique solution $u \in C^{\infty}(\Omega) \cap C^{n+3 / 2-\epsilon}(\bar{\Omega})$. Lee-Melrose [L-M] later improved the regularity to $C^{n+2-\epsilon}(\bar{\Omega})$.

For any domain $\Omega \subset \subset \mathbb{C}^{n}$, it follows from the well-known identity $\operatorname{det}\left[(-\log u)_{\alpha \bar{\beta}}\right]=J(u) / u^{n+1}$ that the following identities are equivalent

$$
B(z)=C_{n} \Leftrightarrow|J(K)|=C_{n} K^{n+2} \Leftrightarrow J\left[\left(\frac{C_{n}}{(n+1)^{n}} K\right)^{-\frac{1}{n+1}}\right]=1 .
$$

Proposition 1.1. Let $\Omega$ be a strictly pseudoconvex domain with smooth boundary. Then the Bergman metric is Kähler-Einstein if and only if $B(z)=(n+$ $1)^{n} \pi^{n} / n$ ! for all $z \in \Omega$.

Proof. It is easy to see that $B(z)$ being a constant implies that the Ricci curvature of the Bergman metric is identical to -1 . We now prove that necessity. Since the Ricci curvature of the Bergman metric tends to -1 as $z$ approaches the boundary $b \Omega$ [C-Y, pp. 510], we have $R_{\alpha \bar{\beta}}=-g_{\alpha \bar{\beta}}$. Therefore $(\log B(z))_{\alpha \bar{\beta}}=0$ on $\Omega$. It follows from [Di, Theorem 2] that $B(z) \rightarrow(n+1)^{n} \pi^{n} / n$ ! as $z \rightarrow b \Omega$. Applying the maximum principle to the pluriharmonic function $\log B(z)$, we have $B(z) \equiv(n+1)^{n} \pi^{n} / n$ !.

A domain $\Omega \subset \mathbb{C}^{n}$ is called complete Reinhardt if $\left(\zeta_{1} z_{1}, \ldots, \zeta_{n} z_{n}\right) \in \Omega$ whenever $\left(z_{1}, \ldots, z_{n}\right) \in \Omega$ and $\left|\zeta_{k}\right| \leq 1,1 \leq k \leq n$.

Proposition 1.2. Let $\Omega \subset \subset \mathbb{C}^{n}$ be a complete Reinhardt domain. Then the Bergman metric of $\Omega$ is Kähler-Einstein with Ricci curvature -1 if and only if $B(z)$ is identical to a constant.

Proof. We need only to prove the necessity. As in the proof of Proposition 1.1, we have $(\log B(z))_{\alpha \bar{\beta}}=0$. Thus $B(z)=\exp (\operatorname{Re} f(z))$ for some holomorphic function $f(z)$ on $\Omega$. Since $B(z)$ is invariant under a $T^{n}$-action, so is $\operatorname{Re} f(z)$. It follows that $f(z)$ is identical to a constant.

\section{$\S 2$. Proof of the main theorem}

Let $\Omega=\left\{z \in \mathbb{C}^{n} ; \rho(z)>0\right\}$ be a strictly pseudoconvex domain with a smooth defining function $\rho$. It follows from the work of Fefferman [Fe1] that the Bergman kernel has the asymptotic expansion

$$
K(z, z)=\frac{\phi(z)}{\rho^{n+1}(z)}+\psi(z) \log \rho(z)
$$

where $\phi, \psi \in C^{\infty}(\bar{\Omega})$ and $\left.\phi\right|_{b \Omega} \neq 0$.

Theorem 2.1. Let $\Omega=\left\{z \in \mathbb{C}^{n} ; \rho>0\right\}$ be a strictly pseudoconvex domain with a smooth defining function $\rho$. If the Bergman metric of $\Omega$ is KählerEinstein, then the coefficient of the logarithmic term in Fefferman's expansion (2.1) vanishes to infinite order at $b \Omega$, i.e., $\psi=O\left(\rho^{k}\right)$ for any $k>0$.

To prove Theorem 2.1, we will need the following Lemma. 
Lemma 2.2. Let $f_{0}(t), \ldots, f_{m}(t) \in C^{\infty}(-\epsilon, \epsilon)$ for some $\epsilon>0$. If

$$
f_{0}(t)+f_{1}(t) \log t+\ldots+f_{m}(t)(\log t)^{m}=0
$$

for all $t \in(0, \epsilon)$, then each $f_{j}(t), 0 \leq j \leq m$, vanishes to infinite order at 0 .

Proof of Theorem 2.1. Assuming Lemma 2.2 for a moment, we now prove Theorem 2.1. It follows from direct calculation that,

$$
\begin{aligned}
K_{\alpha}(z, z)=\rho^{-n-2} & \left(-(n+1) \phi \rho_{\alpha}+\phi_{\alpha} \rho+\psi \rho_{\alpha} \rho^{n+1}+\psi_{\alpha} \rho^{n+2} \log \rho\right), \\
K_{\bar{\beta}}(z, z)=\rho^{-n-2} & \left(-(n+1) \phi \rho_{\bar{\beta}}+\phi_{\bar{\beta}} \rho+\psi \rho_{\bar{\beta}} \rho^{n+1}+\psi_{\bar{\beta}} \rho^{n+2} \log \rho\right), \\
K_{\alpha \bar{\beta}}(z, z)=\rho^{-n-3} & \left((n+1)(n+2) \phi \rho_{\alpha} \rho_{\bar{\beta}}\right. \\
& -(n+1)\left(\phi_{\alpha} \rho_{\bar{\beta}}+\rho_{\alpha} \phi_{\bar{\beta}}+\phi \rho_{\alpha \bar{\beta}}\right) \rho+\phi_{\alpha \bar{\beta}} \rho^{2} \\
& -\psi \rho_{\alpha} \rho_{\bar{\beta}} \rho^{n+1}+\left(\psi_{\alpha} \rho_{\bar{\beta}}+\rho_{\alpha} \psi_{\bar{\beta}}+\psi \rho_{\alpha \bar{\beta}}\right) \rho^{n+2} \\
& \left.+\psi_{\alpha \bar{\beta}} \rho^{n+3} \log \rho\right) .
\end{aligned}
$$

By (1.1) and Proposition 1.1, we have

$$
\operatorname{det}\left(\begin{array}{cc}
K & K_{\bar{\beta}} \\
K_{\alpha} & K_{\alpha \bar{\beta}}
\end{array}\right)=\frac{(n+1)^{n} \pi^{n}}{n !} K^{n+2} .
$$

After substituting (2.1) and (2.3) into (2.4) and then multiplying both sides of (2.4) by $\rho^{n^{2}+4 n+2}$ (to cancel the negative powers of $\rho$ ), we compare the coefficients of the powers of $\log \rho$. It is easy to see that the logarithmic term $(\log \rho)^{n+2}$ appears only on the right hand side of equation (2.4). It then follows from Lemma 2.2 that $\psi$ vanishes to infinite order at $b \Omega$.

Proof of Lemma 2.2. Proving by contradiction, we suppose that some $f_{j}(t), 0 \leq$ $j \leq m$, vanishes only to finite order. Let $k$ be the minimum of the vanishing order of $f_{j}, 0 \leq j \leq m$. Write $f_{j}(t)=a_{j} t^{k}+O\left(t^{k+1}\right)$ and let $j_{0}$ be the largest index $j$ such that $a_{j} \neq 0$. Multiplying both sides of $(2.2)$ by $t^{-k}(\log t)^{-j_{0}}$, we obtain

$$
\sum_{j=0}^{j_{0}-1} f_{j}(t) t^{-k}(\log t)^{j-j_{0}}+\left(a_{j_{0}}+O(t)\right)+\sum_{j=j_{0}+1}^{m} f_{j}(t) t^{-k}(\log t)^{j-j_{0}}=0
$$

Letting $t \rightarrow 0^{+}$, we obtain that the first and the third terms in (2.5) tend to 0 . (Note that the first term in (2.5) does not appear if $j_{0}=0$.) Thus $a_{j_{0}}=0$. This contradicts to our assumption.

Proof of the Main Theorem. According to [Fe2], We may choose a defining function $\rho$ of $\Omega$ such that $J(\rho)=1+O\left(\rho^{3}\right)$. By Theorem 2.1, the logarithmic coefficient $\psi(z)=O\left(\rho^{k}\right)$ for any $k>0$. It follows from the work of BurnsGraham (a proof was given in [G1, pp. 129], where the result was credited to 
Burns. See also [M, pp. 23]) that if $\Omega \subset \subset \mathbb{C}^{2}$ and $\psi=O\left(\rho^{2}\right)$, then $\Omega$ is locally spherical. (Recall that a smooth hypersurface in $\mathbb{C}^{n}$ is called locally spherical if it is locally CR equivalent to the unit sphere $S^{2 n-1}$. See [B-S] for related results in this direction.) Recently, it was proved by Chern and Ji [C-J] that a simply connected domain in $\mathbb{C}^{n}$ with smooth locally spherical boundary is necessary biholomorphic to the unit ball.

\section{$\S 3$. Further Remarks}

(1) If the domain $\Omega$ in the main theorem is assumed to be complete Reinhardt, then the strictly pseudoconvex condition can be reduced to pseudoconvex of finite type. This is true because the Kähler-Einstein condition in this case implies that the domain must be strictly pseudoconvex. We sketch the proof as follows.

It follows from the arguments in $[\mathrm{Fu}]$ that if $\Omega \subset \subset \mathbb{C}^{2}$ is a smoothly bounded pseudoconvex Reinhardt domain and $p=\left(p_{1}, p_{2}\right)$ is a boundary point of type $2 m$, then

$$
\lim _{\substack{z \rightarrow p \\ n . t .}} B_{\Omega}(z)= \begin{cases}B_{\Omega_{m}}((0,0)), & p_{1} p_{2}=0 \\ B_{T_{m}}((0,0)), & p_{1} p_{2} \neq 0\end{cases}
$$

where $\Omega_{m}=\left\{\left(z_{1}, z_{2}\right) ; \operatorname{Re} z_{1}+\left|z_{2}\right|^{2 m}<1\right\}$ and $T_{m}=\left\{\left(z_{1}, z_{2}\right) ; \operatorname{Re} z_{1}+\left(\operatorname{Re} z_{2}\right)^{2 m}<\right.$ $1\}$. It is known (see $[\mathrm{G}-\mathrm{K}]$ ) that

$$
B_{\Omega_{m}}((0,0))=\frac{2 \pi^{2}(2 m+1)(m+2)}{(m+1)^{2}} .
$$

The computations in $[\mathrm{Fu}]$ yield that

$$
B_{T_{m}}((0,0))=\frac{2 \pi^{2}(2 m+1) B_{m}}{m A_{m}^{2}}
$$

where

$$
\begin{gathered}
A_{m}=\Gamma\left(2+\frac{1}{m}\right) \int_{-\infty}^{+\infty} \frac{1}{h_{m}(s)} d s, \quad B_{m}=\Gamma\left(2+\frac{2}{m}\right) \int_{-\infty}^{+\infty} \frac{s^{2}}{h_{m}(s)} d s \\
h_{m}(s)=\int_{-\infty}^{+\infty} e^{-2 s t-t^{2 m}} d t
\end{gathered}
$$

Now if the Bergman metric is Kähler-Einstein, by (the proofs of) Proposition 1.1 and $1.2, B(z)=9 \pi^{2} / 2$. It is obvious that $B_{\Omega_{m}}((0,0)) \leq 9 \pi^{2} / 2$ and the equality holds only when $m=1$. It is easy to see that $\lim _{m \rightarrow \infty} B_{T_{m}}((0,0))=4 \pi^{2}$. However, the computations of $A_{m}$ and $B_{m}$ are rather complicate. We are able to prove with aid of computer that $B_{T_{m}}((0,0)) \leq 9 \pi^{2} / 2$ and the equality holds only when $m=1$. 
(2) A theorem of $\mathrm{Lu}[\mathrm{L}]$ states that a bounded domain in $\mathbb{C}^{n}$ (without the simply connected condition) having a complete Bergman metric with constant holomorphic sectional curvature is necessary biholomorphic to a ball. In light of this result, it would be interesting to see if the simply connected condition in the main theorem can be dropped.

(3) It was asked by D'Angelo (see [Fe3, pp. 260]) whether there are relations between the solution to Monge-Ampére equation (MA) and the Bergman kernel of a weakly pseudoconvex domain. Another interesting question asked by D'Angelo and Ramadanov (see [Da], $[R]$ ) is to characterize those smoothly bounded strictly pseudoconvex domains such that the logarithmic coefficient $\psi(z)$ in Fefferman's expansion (2.1) vanishes on the boundary. A counterexample by R. Graham [G2, pp. 153] showed that the order of vanishing in the above-mentioned result of Burns-Graham is sharp, i.e., there are strictly pseudoconvex domains in $\mathbb{C}^{2}$ with real analytic, but non-locally spherical boundary such that $\psi(z)=O(\rho)$. Nevertheless, for a strictly pseudoconvex complete Reinhardt domain in $\mathbb{C}^{2}$, Boichu-Coeuré $[\mathrm{B}-\mathrm{C}]$ and Nakazawa $[\mathrm{N}]$ proved that if $\psi(z)=O(\rho)$, then the domain is biholomorphic to the ball. Similar result for real ellipsoids that are sufficiently closed to the ball was obtained by Hirachi $[\mathrm{H}]$. Hirachi's result, combining with Theorem 2.1, implies that for a real ellipsoid in $\mathbb{C}^{n}$ that is sufficiently closed to the ball, if its Bergman metric is Kähler-Einstein, then it is biholomorphic to the ball.

\section{Acknowledgement}

The first author is indebted to Professors H. Boas, S. Krantz, and E. Straube for their support. He also thanks Professor H.-D. Cao for stimulating discussions. The authors thank Professors S.-Y. Cheng, Robin Graham, Robert Greene, and Shanyu Ji for providing helpful information on the subject.

\section{References}

[BFG] M. Beals, C. Fefferman, and R. Grossman, Strictly pseudoconvex domains in $\mathbb{C}^{n}$, Bull. Amer. Math. Soc. (N.S.) 8 (1983), 125-322.

[B] S. Bergman, The kernel function and conformal mapping, Mathematical Surveys, No. V., Amer. Math. Soc., Providence, R.I., 1970.

[BSY] H. Boas and E. Straube and J. Yu, Boundary limits of the Bergman kernel and metric, Mich. Math. Jour. 42 (1995), 449-461.

[B-C] D. Boichu and G. Coeuré, Sur le noyau de Bergman des domaines de Reinhardt, Invent. Math. 72 (1983), 131-152.

[B-S] D. Burns and S. Shnider, Real hypersurfaces in complex manifolds, Several Complex Variables, Proc. Symp. Pure Math., vol. 30, Amer. Math. Soc., 1977, pp. 141-168.

[C] S. -Y. Cheng, Private communication.

[C-Y] S.-Y. Cheng and S.-T. Yau, On the existence of a complete Kähler metric on noncompact complex manifolds and the regularity of Fefferman's equation, Comm. Pure Appl. Math. 33 (1980), 507-544.

[C-J] S.-S. Chern and S. Ji, On the Riemann mapping theorem, Ann. of Math 144 (1996), 421-439.

[Da] J. D'Angelo, A note on the Bergman kernel, Duke Math. J. 45 (1978), 259-266. 
[Di] K. Diederich, Das Randverhalten der Bergmanschen Kernfunktion und Metrik in streng pseudoconvexen Gebieten, Math. Ann. 187 (1970), 9-36.

[D-O] K. Diederich and T. Ohsawa, An estimate for the Bergman distance on pseudoconvex domains, Ann. Math. 141 (1995), 181-190.

[Fe1] C. Fefferman, The Bergman kernel and biholomorphic mappings of pseudoconvex domains, Invent. Math. 26 (1974), 1-65.

[Fe2] Monge-Ampére equations, the Bergman kernel, and geometry of pseudoconvex domains, Ann. Math. 103 (1976), 395-416.

[Fe3] - Parabolic invariant theory in complex analysis, Adv. in Math. 31 (1979), 131-262.

[Fu] S. Fu, Geometry of Reinhardt domains of finite type in $\mathbb{C}^{2}$, Jour. of Geom. Anal. (to appear).

[G1] R. Graham, Scalar boundary invariants and the Bergman kernel, Lecture notes in mathematics (C. Berenstein, eds.), vol. 1276, Springer-Verlag, 1987, pp. 108-135.

[G2] Higher asymptotics of the complex Monge-Amperé equation, Compositio Math. 64 (1987), 133-155.

[G-K] R. Greene and S. Krantz, Invariants of the Bergman geometry and the automorphism groups of domainsin $\mathbb{C}^{n}$, Geometrical and algebraical aspects in several complex variables (Cetraro, 1989), Mediterranean Press, 1992, pp. 107-136.

[H] K. Hirachi, The second variation of the Bergman kernel of ellipsoids, Osaka J. Math. 30 (1993), 457-473.

[K-Y] S. Krantz and J. Yu, On the Bergman invariant and curvatures of the Bergman metric, Illinois J. Math. 40 (1996), 226-244.

[L-M] J. Lee and R. Melrose, Boundary behaviour of the complex Monge-Ampére equation, Acta Math. 64 (1982), 159-192.

[L] Q.-K. Lu (=K. H. Look), On Kähler manifolds with constant curvature, Acta Math. Sinica 16 (1966), 269-281.

[M-Y] N. Mok and S.-T. Yau, Completeness of the Kähler-Einstein metric on bounded domains and the characterization of domains of holomorphy by curvature conditions, The Mathematical Heritage of Henri Poincaré, Proc. of Symp. in Pure Math., vol. 39, Amer. Math. Soc., 1983, pp. 41-59.

[M] L. de Monvel, Singularity of the Bergman kernel, Complex geometry (G. Komatsu and Y. Sakane, eds.), Lect. notes in Pure and Applied Math.,, vol. 140, 1993, pp. 1329.

[N] N. Nakazawa, Asymptotic expansion of the Bergman kernel for strictly pseudoconvex complete Reinhardt domains in $\mathbb{C}^{2}$, Osaka J. Math. 31 (1994), 291-329.

[O] T. Oshawa, On the completeness of the Bergman metric, Proc. Jap. Acad. Sci., Ser. A 57 (1981), 238-240.

[P] P. Pflug, Various applications of the existence of well-growing holomorphic functions, Functional Analysis, Holomorphy and Approximation Theorey (G. I. Zapata, eds.), Math. Studies 86, North Holland, 1984, pp. 331-337.

[R] I. P. Ramadanov, A characterization of the balls in $\mathbb{C}^{n}$ by means of the Bergman kernel, Compte Rendu de l'Aca. Bul. des Sci. 34 (1981), 927-929.

[Y] S.-T. Yau, Problem Section, Seminar on Differential geometry (S.-T. Yau, eds.), Ann. of Math. Studies, vol. 102, Princeton Univ. Press, 1982, pp. 669-706.

Department of Mathematics, Texas A \& M University, College Station, TX 77843 E-mail address: sfu@math.tamu.edu

Department of Mathematics, University of California, Riverside, CA 92521

E-mail address: wong@math.ucr.edu 